\title{
CRITERIOS DE RESPUESTA PARA DEFINIR ÉXITO EN LA TERAPIA DE PACIENTES HEMOFÍLICOS LLEVADOS A CIRUGÍA REVISIÓN AMPLIADA DE LA LITERATURA (RAL)
}

Claudia Patricia Casas M D*, J heremy Reyes M D**, M aria Helena Solano M D***, Daniel Espinosa M D****, Virginia A bello M D****

\section{Resumen}

La hemofilia, enfermedad congénita de baja prevalencia en la población general, se clasifica en tipos A y B según su deficiencia sea del factor VIII o IX de la coagulación. Desde hace más de 40 años existen preparados plasmáticos de cada uno de los factores y en la actualidad contamos con derivados recombinantes. Los pacientes que requieren intervenciones quirúrgicas son un reto para los diferentes grupos de manejo, porque el estándar de criterios para definir la respuesta a la terapia no está claro. Se realizó esta revisión ampliada de la literatura con el fin de evaluar cuáles son los criterios de respuesta en las diferentes publicaciones. En las principales bases de datos médicos, se recolectaron y tabularon 61 artículos después de aplicar los criterios de inclusión y exclusión, encontrando que múltiples estudios aplicaban diferentes parámetros para evaluar el sangrado como son los niveles de factor de la coagulación, tromboelastografía, tiempo de generación de trombina, reintervención quirúrgica, duración de la hospitalización y evaluación de dolor, con lo cual concluimos que hay heterogeneidad en los parámetros que aplican los autores en relación con los objetivos principal y secundarios de nuestra revisión.

Palabras clave: hemofilia, cirugía, desenlaces.

\section{EVALUATION OF THERAPEUTIC RESPONSE IN HEMOPHILIC PATIENTS REQUIRING SURGICAL PROCEDURE}

\section{Abstract}

Hemophilia is an inherited low prevalence disorder among the general population classified as type A or B according to the deficiency of either coagulation factor VIII or IX. Coagulation factors in plasma prepared have been available for more than forty years, counting with recombinant factor products nowadays. Patients requiring surgical interventions are a challenge for treating physicians for the criteria standards of therapeutic response are not yet clear. This extended literature review was conducted to evaluate the therapeutic response criteria described in different publications. Sixty-one articles were retrieved from the main medical data bases and tabulated after applying the inclusion and exclusion criteria. Findings showed that many studies considered various parameters to monitor bleeding, such as, levels of coagulation factor, thromboelastography, thrombin generation time, surgical reintervention, length of hospital stay and level of pain. Thus, we conclude there is heterogeneity in the parameters authors apply regarding the primary and secondary objectives of our review.

Key words: hemophilia, surgery, outcomes

Fecha recibido: noviembre 26 de 2014 - Fecha aceptado: diciembre I2 de 2014

* Hematóloga, Hospital de San José. Instructor Asociado, Fundación Universitaria de Ciencias de la Salud. Bogotá DC, Colombia.

** Residente II de Hematología, Fundación Universitaria de Ciencias de la Salud. Bogotá DC, Colombia.
*** Jefe del Servicio de Hematología, Hospital de San José. Profesor Titular Fundación Universitaria de Ciencias de la Salud. Bogotá DC, Colombia.

***** Hematólogo Hospital de San José. Instructor Asistente, Fundación Universitaria de Ciencias de la Salud. Bogotá DC, Colombia. 


\section{Introducción}

La hemofilia es una enfermedad congénita ligada al cromosoma $X$ de baja incidencia en la población general. Se clasifica en tipo A y B dependiendo si el déficit del factor de la coagulación es V III O IX.$^{1,2}$ La hemofilia $A$ es la más frecuente, se estima que uno de cada 5.000 a 10.000 nacimientos masculinos la padecerá. 3,4 Presenta unas características clásicas como son sangrado espontáneo 0 excesiva hemorragia secundaria a un traumatismo accidental o por procedimientos invasivos. ${ }^{5,6} \mathrm{El}$ grado de deficiencia del factor se categoriza en leve ( 5 a $40 \%$ de la actividad del factor VIII, tienden a sangrar sólo después de procedimientos quirúrgicos mayores), moderado (1 a $5 \%$ de la actividad del factor V III) y grave (actividad $<1 \%$ ), siendo los últimos sintomáticos por lo regular en forma espontánea ${ }^{7,8}$; hay correlación directa entre los niveles de factor y la gravedad potencial del sangrado., 10

L os hemofílicos llevados a procedimientos quirúrgicos electivos o de urgencia, son un reto para los diferentes grupos multidisciplinarios. ${ }^{11} \mathrm{H}$ ay gran controversia sobre cuál o cuáles son los parámetros más relevantes para evaluar los pacientes a la hora de definir éxito hemostático. ${ }^{12,13} \mathrm{El}$ objetivo de la presente revisión fue valorar los criterios de éxito de respuesta hemostática tenidos en cuenta en los diferentes estudios publicados en la literatura médica mundial.

\section{Metodología}

Se realizó un estudio secundario tipo revisión ampliada de la literatura; la búsqueda de la información se basó en los estudios publicados en las bases de datos de PUBMED, Cochrane y LILACS. Se identificaran aquellos en inglés y español con los siguientes términos de búsqueda hemophilia $A$, hemophilia $B$, coagulation disorder, treat, therap, manage, current, traditional, conventional, healthoutcome, Impact, effect, surgery y bleeding. Se usaron los operadores B ooleanos OR y AND en relación con la combinación de términos MESH. Se incluyeron estudios experimentales, de cohortes, revisiones sistemáticas de la literatura y metaanálisis, series de casos y estudios de casos controles.

Se restringió la búsqueda de la literatura a publicaciones de pacientes con diagnóstico de hemofilia tipo A o B, tratamiento, severidad, tipo de manejo y los factores clínicos y paraclínicos que evalúan la respuesta a la terapia de reposición de factores. Se tomó como referencia temporal desde 1970 hasta 2012. Se excluyeron estudios con enfermedades y trastornos de la coagulación diferentes a hemofilia tipo $A$ y $B$, aquellos cuya publicación primaria no fuera en inglés o español y los relativos a pacientes con hemofilia adquirida (Figura 1).

Cada publicación se incluyó en una tabla de evidencia que tenía en cuenta las siguientes variables: fecha de publicación, título, autores, control de sangrado, días promedio de hospitalización, uso de tromboelastografía (TEG), tiempo de generación de trombina (TGT),

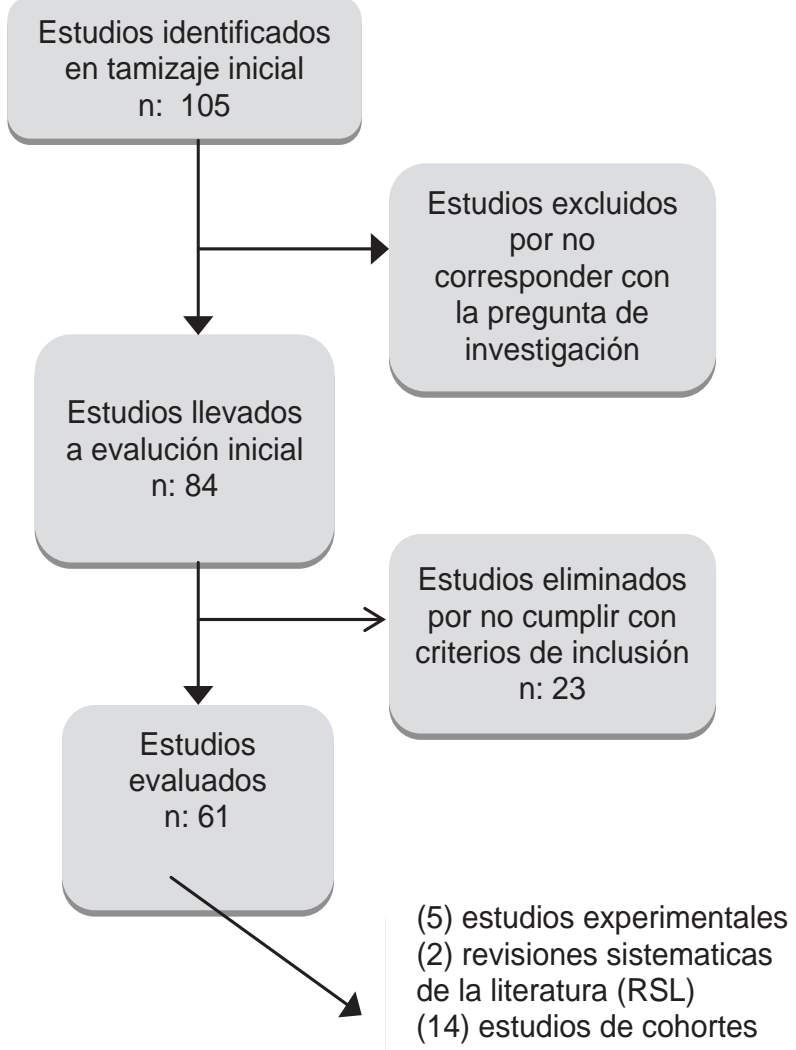

Figura I. Distribución de los estudios evaluados. 
medición de niveles de factor, requerimiento de soporte transfusional, nueva intervenciónn quirúrgica por sangrado, días de aplicación del factor, control del dolor y uso de medidas alternativas para detener el sangrado. Estas variables fueron las categorizadas debido a que son los desenlaces mencionados en los diferentes estudios.

\section{Resultados}

Se encontraron ciento cinco estudios, de los cuales sesenta y uno cumplían con los criterios de inclusión, cinco experimentales, dos revisiones sistemáticas, catorce de cohortes y cuarenta de series de casos. Se real izó una matriz para la clasificación y categorización y se llevó a cabo el análisis en forma independiente por los investigadores.

Se encontró que la mayoría de los estudios reportan niveles de éxito el evados considerados como excelente y bueno ${ }^{14-16},(88 \%)$. Sin embargo, algunos postularon la medición protocolizada de niveles de factores en sangre ${ }^{17,18}$ así como sus propiedades farmacocinéticas, como punto de corte para determinar el éxito o fracaso del control hemostático (Figura 2). ${ }^{19,20}$

Variables como TGE (tromboelastografía) y TGT (tiempo de generación de trombina) se mencionaron como desenlace en $3 \%^{21,22}$ y $1 \%^{23}$ respectivamente. $L$ a dosis de factor usado, se informó en $54 \%$.24,25 U no de los parámetros como fue la medición y control del dolor se reportó en $6 \%{ }^{26,27}$, esta variable tiene impacto en la calidad de vida, control de síntomas y la calidad integral de la atención. ${ }^{28,29}$ Por último $9 \%$ informan requerimiento de soporte transfusional. ${ }^{30-32} \mathrm{M}$ edidas implementadas como la administración de pegamento de fibrina ${ }^{33,34}$, fibrinolíticos como el ácido tranexámi$\mathrm{Co}^{35,36}$ y el ácido epsilon-aminocaproico ${ }^{37,38}$, también fueron valorados en varias investigaciones.

\section{Discusión}

La hemofilia se encuentra dentro de las enfermedades calificadas como huérfanas ${ }^{39}$, dada su baja prevalencia. ${ }^{40-42} \mathrm{La}$ expectativa de vida de los pacientes a principio del siglo XX se limitaba a la edad pediátrica. ${ }^{43,44}$ Después con al advenimiento de los crioprecipitados mejoró la supervivencia ${ }^{45-47}$ pero la la aparición de infecciones por V IH y hepatitis conllevó a un deterioro en estos parámetros. ${ }^{48,49} \mathrm{EI}$ desarrollo de nuevos avances para mejorar el perfil de seguridad de los productos, permitió mejoría de los desenlaces. ${ }^{50-52}$

En la actualidad se dispone de un mayor número de productos para el manejo de esta condición, con un perfil de seguridad y eficacia clínica. ${ }^{53,54}$ El cambio de manejar los eventos a tener la disponibilidad de profilaxis ya sea primaria, secundaria o terciaria, ha llevado a mejoría en cuanto a artropatía ${ }^{55,56}$, calidad de vida e impacto en cambios en los indicadores de ausencia escolar o laboral y acceso a procedimientos quirúrgicos. ${ }^{57,58}$

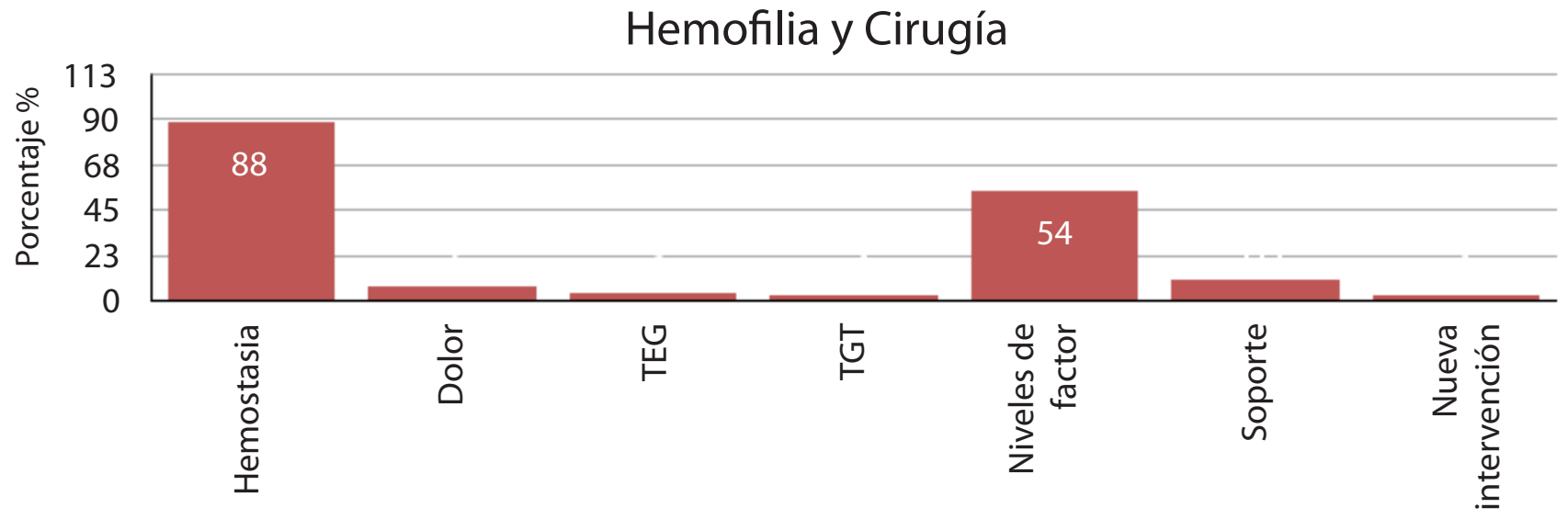

Figura 2. Desenlaces evaluados. 
El desarrollo de la investigación en este grupo poblacional tiene condiciones especiales ya que el número de pacientes es limitado, el estudio en pediatría tiene implicaciones éticas que pueden disminuir la muestra. ${ }^{59} \mathrm{H}$ oy existen adelantos desde el punto de vista de investigación, en los que el rigor investigativo, metodológico y las exigencias de las autoridades sanitarias para la aprobación de nuevos medicamentos ha aumentado en los estándares de investigación.

L uego de la búsqueda y análisis de la información se evidencia un vacío en lo relacionado con la estandarización y cuantificación de los procedimientos de monitoreo, pautas globales para las diferentes poblaciones de pacientes en condición de hemofilia, protocolos de tratamiento de complicaciones y falta de claridad en los determinantes de control hemostático.

Hoy día el plan de manejo de los pacientes con hemofilia es un conjunto que incluye la educación del paciente y su familia, la clasificación en los distintos grupos de acuerdo con los niveles del factor, las complicaciones infecciosas y el desarrollo de inhibidores. Hay gran expectativa acerca de nuevos tratamientos como la inmunomodulación y la terapia génica, pero aun factores como los criterios de respuesta a cada una de las terapias no están estandarizados.

La revisión realizada evaluó los diversos criterios de respuesta usados en una muestra representativa de estudios clínicos, en los que se incluyen pacientes en condición de hemofilia llevados a procedimientos quirúrgicos. Se evidencia la necesidad de investigar más a fondo los factores que intervienen en el control del sangrado después de someter a un paciente hemofílico a una cirugía. Falta estandarizar los lineamientos que deben tener en cuenta y por tanto es necesario realizar más investigaciones al respecto.

\section{Conclusiones}

Existe una marcada heterogeneidad en relación con la estandarización y cuantificación de los procedimientos de monitoreo, tanto clínicos como de laboratorio, pautas globales para las diferentes subpoblaciones de pacientes en condición de hemofilia, protocolos de tratamiento de complicaciones y falta de claridad en los determinantes de control hemostático. Se considera además el logro de encontrar múltiples procesos que deben estandarizarse y evaluarse por separado con la evidencia disponible a la actualidad, más aun al tener la posibilidad de aportar evidencia con proyectos de investigación de al ta calidad que impacten en el futuro de las determinaciones que se tomen en relación con el paciente en condición de hemofilia que sea intervenido quirúrgicamente.

Como no es claro aún si son de mayor relevancia los criterios clínicos vs. los paraclínicos en relación con la toma de decisiones cuando se plantea la posibilidad de control hemostático o éxito hemostático en el paciente hemofílico que es Ilevado a un procedimiento quirúrgico, se requieren más estudios al respecto.

\section{Referencias}

1. Aledort LM. History of haemophilia. Haemophilia. 2007 Dec;13 Suppl 5:1-2.

2. Oldenburg J, Dolan G, Lemm G. Haemophilia care then, now and in the future. Haemophilia. 2009 Jan; 15 Suppl 1:2-7.

3. Stachnik J. Hemophilia: Etiology, complications, and current options in management. Clin Pharm. 2010: 45.

4. Wong T, Recht M. Current options and new developments in the treatment of haemophilia. Drugs. 2011 Feb; 71(3):305-20.

5. Lee CA. The best of times, the worst of times: a story of haemophilia. Clin Med. 2009 Oct; 9(5):453-8.

6. Evatt BL. The tragic history of AIDS in the hemophilia population. 1982-1984. J Thromb Haemost. 2006 Nov; 4(11):2295-301.

7. Mannucci PM. AIDS, hepatitis and hemophilia in the 1980s: memoirs from an insider. J Thromb Haemost. 2003 Oct;1(10):2065-9

8. Chorba TL, Holman RC, Strine TW, Clarke MJ, Evatt BL. Changes in longevity and causes of death among persons with hemophilia A. Am J Hematol. 1994 Feb;45(2):112-21

9. Mannucci PM, Tuddenham EG. The hemophilias from royal genes to gene therapy. N Engl J Med. 2001 Jun 7; 344(23):1773-9.

10. Rodriguez-Merchan EC. Local fibrin glue and chitosan-based dressings in haemophilia surgery. Blood Coagul Fibrinolysis. 2012 Sep; 23(6):473-6.

11. Aznar JA, Marco A, Jiménez-Yuste V, et al. Is on-demand treatment effective in patients with severe haemophilia?. Haemophilia. 2012 Sep;18(5):738-42.

12. Eckhardt CL, Mauser-Bunschoten EP, Peters M, Leebeek FW, et al. Inhibitor incidence after intensive FVIII replacement for surgery in mild and moderate haemophilia A: a prospective national study in the Netherlands. Br J Haematol. 2012 Jun; 157(6):747-52.

13. Batorova A, Holme P, Gringeri A, Richards M, Hermans C, Altisent C, et al Continuous infusion in haemophilia: current practice in Europe. Haemophilia. 2012 Sep; 18(5):753-9.

14. Pérez-Garrido R, Alonso N, Jiménez-Yuste V et al. Efficacy of factor IX Grifols( $\left({ }^{\circledR}\right)$ in surgery: experience of an international multicenter retrospective study. Haemophilia. 2012 Sep; 18(5):e372-3.

15. Holmström M, Tran HT, Holme PA. Combined treatment with APCC (FEIBA®) and tranexamic acid in patients with haemophilia A with inhibitors and in patients with acquired haemophilia A--a two-centre experience. Haemophilia. 2012 Jul; 18(4):544-9.

16. Coppola A, Franchini M, Makris M, Santagostino E, et al. Thrombotic adverse events to coagulation factor concentrates for treatment of patients with haemo- 
philia and von Willebrand disease: a systematic review of prospective studies. Haemophilia. 2012 May;18(3):e173-87.

17. Berntorp E, Astermark J, Baghaei F, et al. Treatment of haemophilia A and B and von Willebrand's disease: summary and conclusions of a systematic review as part of a Swedish health-technology assessment. Haemophilia. 2012 Mar; 18(2):158-65.

18. Zülfikar B, Aydogan G, Salcioglu Z, Oner AF, Kavakli K, et al. Efficacy of FEIBA for acute bleeding and surgical haemostasis in haemophilia A patients with inhibitors: a multicentre registry in Turkey. Haemophilia. 2012 May; 18(3):383-91.

19. Eckhardt CL, van der Bom JG, van der Naald M, et al. Surgery and inhibitor development in hemophilia A: a systematic review. J Thromb Haemost. 2011 Oct; 9(10):1948-58.

20. Rossi M, Jayaram R, Sayeed R. Do patients with haemophilia undergoing cardiac surgery have good surgical outcomes?. Interact Cardiovasc Thorac Surg. 2011 Sep; 13(3):320-31.

21. Mauser-Bunschoten EP, Kleine Budde I, Lopaciuk S, Koopman MM, et al. An ultrapure plasma-derived monoclonal antibody-purified factor IX concentrate (Nonafact $\left.{ }^{\circledR}\right)$, results of phase III and IV clinical studies. Haemophilia. 2011 May; 17(3):439-45.

22. Oldenburg J, Goudemand J, Valentino L, et al. Postauthorization safety surveillance of ADVATE [antihaemophilic factor (recombinant), plasma/albumin-free method] demonstrates efficacy, safety and low-risk for immunogenicity in routine clinical practice. Haemophilia. 2010 Nov; 16(6):866-77.

23. Windyga J, Rusen L, Gruppo R, et al. BDDrFVIII (Moroctocog alfa [AF-CC]) for surgical haemostasis in patients with haemophilia A: results of a pivotal study. Haemophilia. 2010 Sep 1; 16(5):731-9.

24. Martinowitz U, Luboshitz J, Bashari D, et al. Stability, efficacy, and safety of continuously infused sucrose-formulated recombinant factor VIII (rFVIII-FS) during surgery in patients with severe haemophilia. Haemophilia. 2009 May; 15(3):676-85.

25. Giangrande PL, Wilde JT, Madan B, et al. Consensus protocol for the use of recombinant activated factor VII [eptacog alfa (activated); NovoSeven] in elective orthopaedic surgery in haemophilic patients with inhibitors. Haemophilia. 2009 Mar; 15(2):501-8.

26. Négrier C, Shapiro A, Berntorp E, et al. Surgical evaluation of a recombinant factor VIII prepared using a plasma/albumin-free method: efficacy and safety of Advate in previously treated patients. Thromb Haemost. 2008 Aug; 100(2):217-23.

27. Schwarz KB, Zellos A, Stamato L, et al. Percutaneous liver biopsy in hemophiliac children with chronic hepatitis C virus infection. J Pediatr Gastroenterol Nutr. 2008 Apr; 46(4):423-8.

28. Miles J, Rodríguez-Merchán EC, Goddard NJ. The impact of haemophilia on the success of total hip arthroplasty. Haemophilia. 2008 Jan; 14(1):81-4.

29. Correa ME, Annicchino-Bizzacchi JM, Jorge J Jr, et al. Clinical impact of oral health indexes in dental extraction of hemophilic patients. J Oral Maxillofac Surg. 2006 May; 64(5):785-8.

30. Franchini M, Rossetti G, Tagliaferri A, et al. Dental procedures in adult patients with hereditary bleeding disorders: 10 years experience in three Italian Hemophilia Centers. Haemophilia. 2005 Sep; 11(5):504-9.

31. Türkmen C, Zülflkar B, Taşer O, et al. Radiosynovectomy in hemophilic synovitis: correlation of therapeutic response and blood-pool changes. Cancer Biother Radiopharm. 2005 Jun; 20(3):363-70.

32. Soroa VE, del Huerto Velázquez Espeche M, Giannone C, et al. Effects of radiosynovectomy with p-32 colloid therapy in hemophilia and rheumatoid arthritis. Cancer Biother Radiopharm. 2005 Jun; 20(3):344-8.

33. Lee AP, Boyle CA, Savidge GF, Fiske J. Effectiveness in controlling haemorrhage after dental scaling in people with haemophilia by using tranexamic acid mouthwash. Br Dent J. 2005 Jan 8; 198(1):33-8.

34. Karaman MI, Zulfikar B, Caskurlu T, Ergenekon E. Circumcision in hemophilia: a cost-effective method using a novel device. J Pediatr Surg. 2004 Oct; 39(10):1562-4.

35. Stieltjes N, Altisent C, Auerswald G, et al. Continuous infusion of B-domain deleted recombinant factor VIII (ReFacto) in patients with haemophilia A undergoing surgery: clinical experience. Haemophilia. 2004 Sep; 10(5):452-8.

36. Rodriguez-Merchan EC, Wiedel JD, Wallny T, et al. Elective orthopaedic surgery for inhibitor patients. Haemophilia. 2003 Sep; 9(5):625-31.

37. Hoots WK, Leissinger C, Stabler S, et al. Continuous intravenous infusion of a plasma-derived factor IX concentrate (Mononine) in haemophilia B. Haemophilia. 2003 Mar; 9(2):164-72.
38. Giangrande PL; KOGENATE Bayer Study Group. Safety and efficacy of KOGENATE Bayer in previously untreated patients (PUPs) and minimally treated patients (MTPs). Haemophilia. 2002 Mar; 8 Suppl 2:19-22.

39. Ragni MV, Pasi KJ, White GC, Giangrande PL, et al. Use of recombinant factor IX in subjects with haemophilia B undergoing surgery. Haemophilia. 2002 Mar; 8(2):91-7.

40. Smith OP. Recombinant factor VIIa in the management of surgery and acute bleeding episodes in children with haemophilia and high-responding inhibitors. Pathophysiol Haemost Thromb. 2002; 32 Suppl 1:22-5.

41. Scharrer I, Brackmann HH, Sultan Y, et al. Efficacy of a sucrose-formulated recombinant factor VIII used for 22 surgical procedures in patients with severe haemophilia A. Haemophilia. 2000 Nov; 6(6):614-8.

42. MacKinlay N, Taper J, Renisson F, et al. Cardiac surgery and catheterization in patients with haemophilia. Haemophilia. 2000 Mar;6(2):84-8.

43. Bollard CM, Teague LR, Berry EW, Ockelford PA. The use of central venous catheters (portacaths) in children with haemophilia. Haemophilia. 2000 Mar;6(2):66-70.

44. Tagariello G, Davoli PG, Gajo GB, et al. Safety and efficacy of high-purity concentrates in haemophiliac patients undergoing surgery by continuous infusion. Haemophilia. 1999 Nov; 5(6):426-30.

45. Scharrer I. Recombinant factor VIIa for patients with inhibitors to factor VIII or IX or factor VII deficiency. Haemophilia. 1999 Jul; 5(4):253-9.

46. Rochat C, McFadyen ML, Schwyzer R, et al. Continuous infusion of intermediate-purity factor VIII in haemophilia A patients undergoing elective surgery. Haemophilia. 1999 May; 5(3):181-6.

47. Shapiro AD, Gilchrist GS, Hoots WK, et al. Prospective, randomised trial of two doses of rFVIIa (NovoSeven) in haemophilia patients with inhibitors undergoing surgery. Thromb Haemost. 1998 Nov; 80(5):773-8.

48. Négrier C, Menart C, Attali O, et al. Evaluation of coagulation equilibrium at baseline and during factor VIII and factor IX replacement in haemophiliacs. Blood Coagul Fibrinolysis. 1998 Mar; 9 Suppl 1:S135-41.

49. Santagostino E, Gringeri A, Muça-Perja M, et al. A prospective clinical trial of implantable central venous access in children with haemophilia. Br J Haematol. 1998 Sep; 102(5):1224-8.

50. F Gordon, P Mistry, C Sabin, and C Lee. Outcome of orthotopic liver transplantation in patients with haemophilia. Gut. May 1998; 42(5): 744-9.

51. Djulbegovic B, Marasa M, Pesto A, et al. Safety and efficacy of purified factor IX concentrate and antifibrinolytic agents for dental extractions in hemophilia B. Am J Hematol. 1996 Feb; 51(2):168-70.

52. Waly NG. Local antifibrinolytic treatment with tranexamic acid in hemophilic children undergoing dental extractions. Egypt Dent J. 1995 Jan;41(1):961-8.

53. Pearce MS, Smith MA, Savidge GF. Supramalleolar tibial osteotomy for haemophilic arthropathy of the ankle. J Bone Joint Surg Br. 1994 Nov; 76(6):947-50.

54. Santagostino E, Mannucci PM, Gringeri A, et al. Markers of hypercoagulability in patients with hemophilia B given repeated, large doses of factor IX concentrates during and after surgery. Thromb Haemost. 1994 Jun; 71(6):737-40.

55. Goldsmith JC, Kasper CK, Blatt PM, et al. Coagulation factor IX: successful surgical experience with a purified factor IX concentrate. Am J Hematol. 1992 Jul;40(3):210-5.

56. Erken EH. Radiocolloids in the management of hemophilic arthropathy in children and adolescents. Clin Orthop Relat Res. 1991 Mar; (264):129-35.

57. Forbes CD, Barr RD, Reid G, Thomson C, et al. Tranexamic acid in control of haemorrhage after dental extraction in haemophilia and Christmas disease. $\mathrm{Br}$ Med J. 1972; 2(5809): 311-3.

58. Simpson E, Lin Y, Stanworth S, et al. Recombinant factor VIIa for the prevention and treatment of bleeding in patients without haemophilia. Cochrane Database Syst Rev. 2012 Mar 14;3: CD005011.

59. Scharrer I. Recombinant factor VIIa for patients with inhibitors to factor VIII or IX or factor VII deficiency. Haemophilia. 1999 Jul; 5(4):253-9.

60. Négrier C, Menart C, Attali O, et al. Evaluation of coagulation equilibrium at baseline and during factor VIII and factor IX replacement in haemophiliacs. Blood Coagul Fibrinolysis. 1998 Mar; 9 Suppl 1:S135-41.

61. Shapiro AD, Gilchrist GS, Hoots WK, et al. Prospective, randomised trial of two doses of rFVIIa (NovoSeven) in haemophilia patients with inhibitors undergoing surgery. Thromb Haemost. 1998 Nov; 80(5):773-8. 